J Ästhet Chir 2020 • 13:70-76

https://doi.org/10.1007/s12631-020-00219-9

Online publiziert: 6. April 2020

(c) Springer Medizin Verlag GmbH, ein Teil von Springer Nature 2020

Die Berufsausübungsgemeinschaft (kurz: BAG; früher vornehmlich als Gemeinschaftspraxis bezeichnet) ist die häufigste Form der (ambulanten) ärztlichen Kooperation. Daher ergeben sich viele wichtige Fragen: Was genau ist eine Berufsausübungsgemeinschaft? Welche rechtlichen Grundsätze gelten für sie? Und wie sollte sie gesellschaftsvertraglich aufgestellt sein, um den individuellen Belangen ihrer Gesellschafter Rechnung zu tragen? Diese Fragen sollen nachstehend beleuchtet werden, wobei zunächst zum Verständnis der Begrifflichkeiten die Berufsausübungsgemeinschaft von den übrigen ärztlichen Kooperationsformen abgegrenzt wird und sodann die Sonderformen der Berufsausübungsgemeinschaft erläutert werden. Nachfolgend wird in Grundzügen dargestellt werden, was eine Berufsausübungsgemeinschaft rechtlich ausmacht und von anderen Kooperationsformen abhebt. Sodann wird im Einzelnen dargestellt, auf welche Aspekte bei der Gestaltung des Gesellschaftsvertrages besonderes Augenmerk zu legen ist und welche Gestaltungsmöglichkeiten insoweit bestehen.

\section{Die verschiedenen Gemein- schaftsformen}

In einer Praxisgemeinschaft teilen sich mehrere Ärzte - bei ansonsten selbstständiger Berufsausübung - vornehmlich gemeinsame Räumlichkeiten, unter Umständen auch nichtärztliches Personal und/oder medizinische Geräte. Dies

Aus Gründen der vereinfachten Lesbarkeit und Darstellung wird vorliegend anstelle der vollständigen Verwendung der männlichen und weiblichen Form allgemein ausschließlich die männliche Form genutzt.

Vera Keisers

Kanzlei am Ärztehaus, Münster, Deutschland

\title{
Rechtliche Grundlagen der Berufsausübungsgemeinschaft
}

dient v. a. der Einsparung von Kosten, weil nicht jeder eine eigene volle Ausstattung und separate Räumlichkeiten benötigt. Ähnlich ist es in der reinen Apparate- oder Gerätegemeinschaft, in der besonders teure Gerätschaften nicht von jedem Arzt angeschafft werden, sondern zwei oder meist mehrere Ärzte sich diese teilen.

Die Berufsausübungsgemeinschaft hingegen ist eine deutlich engere Verbindung. Die Ärzte teilen neben den Räumen und dem nichtärztlichen sowie ärztlichen Personal und den Gerätschaften auch alle weiteren Kosten und Risiken. Sie führen auch eine gemeinsame Patientenkartei, was außerhalb einer Berufsausübungsgemeinschaft strikt untersagt ist. Eine reine Praxisgemeinschaft muss streng trennen, was die Behandlung der Patienten angeht und auch hinsichtlich des Personals: Am Empfang muss unterschieden werden, um wessen Patienten es sich handelt. Patienten des einen Arztes der Praxisgemeinschaft dürfen aus Datenschutzgründen nicht vom alleinigen Personal des anderen Arztes „bedient" werden. Auch die Behandlungsverträge werden in einer Berufsausübungsgemeinschaft nicht mit einem einzelnen Arzt, sondern in der Regel mit der Berufsausübungsgemeinschaft geschlossen. Dies ermöglicht (bei Einverständnis des Patienten) auch eine Behandlung durch einen der anderen Ärzte der Berufsausübungsgemeinschaft, ohne dass hierzu ein gesonderter Behandlungsvertrag erforderlich wäre.

Exkurs Sonderformen der Berufsausübungsgemeinschaft. Eine besondere Ausgestaltung der Berufsausübungsgemeinschaft stellen die überörtliche Berufsausübungsgemeinschaft und die Teil-
Berufsausübungsgemeinschaft (Teil-BAG) dar. Während das „Normalbild“ einer Berufsausübungsgemeinschaft eine örtliche Berufsausübungsgemeinschaft ist, also eine Berufsausübungsgemeinschaft mit nur einem einzigen Praxisstandort, kann eine Berufsausübungsgemeinschaft durchaus auch mit mehreren Standorten betrieben werden. Für eine solche überörtliche Berufsausübungsgemeinschaft gelten allerdings besonders in der vertragsärztlichen Versorgung strenge Vorgaben dazu, in welchem Umfang Ärzte an welchem Standort tätig werden dürfen bzw. müssen. Insbesondere muss in der vertragsärztlichen Versorgung jeder Arzt überwiegend an dem Standort tätig werden, an dem er seine vertragsärztliche Zulassung hat.

Eine Teil-BAG bezeichnet eine Berufsausübungsgemeinschaft, in der die Mitglieder ausschließlich bestimmte Leistungen erbringen, während sie weitere Leistungen außerhalb dieser TeilBAG erbringen.

\section{Es gilt abzugrenzen \\ - Berufsausübungsgemeinschaft (ört- liche oder überörtliche) bzw. Teil- Berufsausübungsgemeinschaft - Praxisgemeinschaft \\ - Apparate- oder Gerätegemeinschaft}

\section{Grundlegende Regelungen für die Berufsausübungsgemein- schaft}

\section{Zulassungsrecht}

Sollen von der Berufsausübungsgemeinschaft vertragsärztliche Leistungen erbracht werden, bedarf eine Berufsaus- 
übungsgemeinschaft (anders als die Praxisgemeinschaft oder Apparategemeinschaft) der vorherigen Genehmigung durch die zuständigen Zulassungsgremien der jeweiligen Kassenärztlichen Vereinigung. Dies gilt sowohl für die örtliche wie auch die überörtliche Berufsausübungsgemeinschaft als auch für die Teil-BAG.

Neben dieser Genehmigung zur gemeinsamen Ausübung vertragsärztlicher Tätigkeit (sog. BAG-Genehmigung) bedarf zudem jeder in der Berufsausübungsgemeinschaft tätige Arzt einer eigenen vertragsarztrechtlichen Grundlage zur Erbringung und Abrechnung vertragsärztlicher Leistungen.

Im Hinblick auf den Erhalt und die wunschgemäße Nutzungsmöglichkeit vorhandener Zulassungen genießt die Berufsausübungsgemeinschaft eine zulassungsrechtliche Privilegierung. Denn im Rahmen der Nachbesetzung einer vertragsärztlichen Zulassung eines Arztes, der Gesellschafter einer Berufsausübungsgemeinschaft (gewesen) ist, sind bei einer Nachbesetzung innerhalb der Berufsausübungsgemeinschaft die Interessen der verbleibenden Mitgesellschafter bevorzugt zu berücksichtigen. Faktisch handhaben die Zulassungsausschüsse dies in der Weise, dass sie unter mehreren Bewerbern die $\mathrm{Zu}$ lassung demjenigen erteilen, den der oder die andere(n) Gesellschafter als Wunschnachfolger benennt/benennen, auch wenn nach den weiteren gesetzlichen Auswahlkriterien andere Bewerber vorzugswürdig wären.

\section{Außenhaftung}

Auch haftungsrechtlich steht eine Berufsausübungsgemeinschaft gegenüber Dritten als Einheit da. Jeder Gesellschafter haftet für die Verbindlichkeiten der Gesellschaft im Außenverhältnis persönlich, es sei denn, es wird die Rechtsform der Partnerschaftsgesellschaft nach dem Partnerschaftsgesellschaftsgesetz (PartGG) gewählt. Diese muss in das Partnerschaftsregister eingetragen werden mit der Folge, dass im Verhältnis nach außen - also zwischen Gesellschaft und Patient - neben der Gesellschaft selbst nur derjenige Arzt persönlich haftet, der den Patienten behandelt hat. (Eine weitere Beschränkung der Haftung, wie sie in anderen Berufszweigen über die Rechtsform der Partnerschaftsgesellschaft mit beschränkter Berufshaftung möglich ist, ist bei Ärzten ausgeschlossen.) In der Rechtsform einer Gesellschaft bürgerlichen Rechts (GbR), die nach wie vor die ganz überwiegend übliche Rechtsform bei Berufsausübungsgemeinschaften ist, haften alle Gesellschafter als Gesamtschuldner persönlich. Das bedeutet: Ein vermeintlich fehlerhaft behandelter Patient kann jeden der Gesellschafter auf Schadensersatz in Anspruch nehmen, egal ob dieser selbst ihn behandelt hat oder nicht.

\section{Achtung}

Im Hinblick auf diese gesamtschuldnerische Haftung nach außen ist v. a. auch für solche Kooperationen Vorsicht geboten, die rechtlich gesehen gar keine Berufsausübungsgemeinschaft sind und dies auch gar nicht sein wollen. Denn auch wenn lediglich eine Praxisgemeinschaft oder Apparategemeinschaft betrieben wird oder sogar in einer Einzelpraxis nur ein (oder mehrere) Arzt/Ärzte als Angestellte(r) beschäftigt wird/werden, kann es durch den Außenauftritt der Praxis passieren, dass eine Haftung wie in der Berufsausübungsgemeinschaft entsteht: Vermitteln etwa die Homepage oder gemeinsames Briefpapier, das Praxisschild, die Terminzettel oder Werbemittel den Eindruck, dass es sich um eine Berufsausübungsgemeinschaft handelt, kann der Patient oder sonstige Gläubiger wie der Vermieter oder Lieferanten sich unter Umständen hierauf berufen und die anderen (ggf. sogar nur angestellten) Ärzte gleichermaßen voll in Haftung nehmen, als wären diese Gesellschafter (sog. Rechtsscheinhaftung).

\section{Beachte}

Wer nach außen auftritt wie eine Berufsausübungsgemeinschaft, der haftet unter Umständen auch wie eine.
Die Haftung im Innenverhältnis hingegen, also die Frage, zu welchem Anteil die Gesellschafter im Verhältnis untereinander welche Verbindlichkeiten tragen, kann auch in der Rechtsform der GbR im Gesellschaftsvertrag abweichend von der Außenhaftung geregelt werden. So kann etwa für Verbindlichkeiten aufgrund von Behandlungsfehlern geregelt werden, dass im Innenverhältnis zwischen den Gesellschaftern nur der behandelnde Arzt alleine hierfür aufzukommen hat (soweit nicht ohnehin die Berufshaftpflichtversicherung einsteht).

\section{Der Gesellschaftsvertrag}

Rechtliche Grundlage einer Berufsausübungsgemeinschaft (egal ob als GbR oder als PartG) ist der Gesellschaftsvertrag, der gelegentlich auch als „Vertrag über eine Berufsausübungsgemeinschaft" oder (von der früher gebräuchlichen Bezeichnung) „Gemeinschaftspraxisvertrag" bezeichnet wird. Bei einer Berufsausübungsgemeinschaft, in der vertragsärztliche Leistungen erbracht werden sollen, ist das Vorliegen eines solchen schriftlichen Gesellschaftsvertrages im Rahmen des Genehmigungsverfahrens der gemeinsamen Ausübung vertragsärztlicher Tätigkeit grundsätzlich zwingend erforderlich. Doch auch wenn ausschließlich privatärztliche Leistungen erbracht werden, sodass lediglich eine Anzeigepflicht bei der Ärztekammer besteht, ist es zur Rechtsklarheit aller Gesellschafter dringend zu empfehlen, einen schriftlichen Gesellschaftsvertrag zu schließen.

Die Ausarbeitung eines solchen Gesellschaftsvertrages bedarf großer Sorgfalt und sollte daher immer von in dieser Spezialmaterie erfahrenen Medizinrechtlern übernommen werden. Besondere Vorsicht gilt bei der Verwendung von Mustern und Vorlagen, da diese immer nur eine denkbare Grundkonstellation betreffen und nie die Feinheiten und unterschiedlichen Interessenlagen des Einzelfalles berücksichtigen (können). So macht schon die Frage, ob es zwei oder mehrere Gesellschafter geben soll, einen bedeutenden Unterschied sowohl in der Entscheidungsfindung wie auch in den Regelungen zum Ausscheiden eines 
Gesellschafters. Auch sind unterschiedliche Interessenlagen der Gesellschafter von großer Bedeutung. Ist der eine Arzt z. B. ein Mittfünfziger, der $65 \mathrm{~h}$ pro Woche arbeitet, aber beabsichtigt, in den nächsten Jahren seine Tätigkeit zu beenden, und der andere Arzt z.B. eine alleinerziehende Mutter, die absehbar innerhalb der nächsten Jahre nicht mehr als $25 \mathrm{~h}$ pro Woche wird arbeiten können, sind in der Vertragsgestaltung ganz andere Überlegungen anzustellen, als wenn etwa beide Ärzte am Beginn ihres Berufslebens stehen und beabsichtigen, beide für die nächsten 30 Jahre voll $\mathrm{zu}$ arbeiten.

In jedem Falle sind im Rahmen eines Gesellschaftsvertrages unter anderem insbesondere die nachfolgend aufgeführten Punkte zu regeln.

\section{Vermögensbeteiligung (Gesellschaftsanteil)}

Wesentlicher Bestandteil eines Gesellschaftsvertrages ist die Beteiligung der Gesellschafter einerseits am materiellen und andererseits am immateriellen Gesellschaftsvermögen. Während das materielle Vermögen v. a. aus dem Praxisinventar einschließlich der Gerätschaften (und bei Eigentumsimmobilien: aus dem Grundstückseigentum bezüglich der Praxisimmobilie) besteht, macht einen Großteil des immateriellen Vermögens der sog. „Goodwill“ aus, also die Möglichkeit, mittels des bestehenden Patientenstammes in Zukunft Umsätze zu generieren. Die Beteiligung der Gesellschafter an materiellem und immateriellem Vermögen kann voneinander abweichen, d. h. die prozentuale Beteiligung am einen muss nicht gleich hoch sein wie die am anderen. Es gibt sogar die zulassungsrechtlich beanstandungsfreie Möglichkeit, lediglich mit einem geringen Anteil am immateriellen Vermögen und gleichzeitig überhaupt nicht am materiellen Vermögen beteiligt zu sein. Auch hier gelten aber - auch bei rein privatärztlich tätigen Praxen in ganz besonderem Maße die unten stehenden Ausführungen (s. „Exkurs Scheinselbstständigkeit“).

J Ästhet Chir 2020 • 13:70-76 https://doi.org/10.1007/s12631-020-00219-9

(c) Springer Medizin Verlag GmbH, ein Teil von Springer Nature 2020

\section{Keisers}

\section{Rechtliche Grundlagen der Berufsausübungsgemeinschaft}

\section{Zusammenfassung}

In der Berufsausübungsgemeinschaft werden nicht nur die Praxisräumlichkeiten, die Praxiseinrichtung und das nichtärztliche Personal von zwei oder mehreren Ärzten gemeinschaftlich genutzt, sondern es kann auch ärztliches Personal gemeinsam beschäftigt werden, und in der Regel laufen zudem sämtliche Einnahmen und Ausgaben gemeinschaftlich über die Praxis. Nach außen bildet die Berufsausübungsgemeinschaft eine rechtliche Einheit, was sich auch in der gemeinsamen Haftung der Gesellschafter niederschlägt. Zum Schutz dieser Einheit darf die Berufsausübungsgemeinschaft auch im Rahmen der Nachbesetzung der Zulassung eines ausscheidenden Gesellschafters über den Nachfolger mitbestimmen, wenn die Zulassung innerhalb der Berufsausübungsgemeinschaft nachbesetzt wird. Im Rahmen der Gestaltung des Gesellschaftsvertrages ist insgesamt große Sorgfalt und in vielerlei Hinsicht auch Vorsicht geboten. Zudem ist immer die individuelle Situation der Gesellschaft und der einzelnen Gesellschafter maßgeblich zu berücksichtigen. Auch die örtliche Versorgungssituation im Hinblick auf die Bedarfsplanung hat bedeutenden Einfluss, $v$. a. auf Regelungen zum Ausscheiden eines Gesellschafters. Die hier aufgezeigten Teilaspekte der Vertragsgestaltung und die jeweiligen Regelungsmöglichkeiten können angesichts der Komplexität der Materie hier nur einen kleinen Ausschnitt darstellen. Mit der Gestaltung eines ärztlichen Gesellschaftsvertrages sollte in Anbetracht der rechtlichen Schnittmengen aus Gesellschaftsrecht, ärztlichem Berufsrecht und ggf. Zulassungsrecht ein Rechtsanwalt beauftragt werden, der über die entsprechende Erfahrung und Expertise verfügt.

\section{Schlüsselwörter}

Gründung/Einstieg/Übernahme einer Berufsausübungsgemeinschaft · Gestaltung des Gesellschaftsvertrages („Gemeinschaftspraxisvertrag") · Gewinnverteilung · Interessengerechte Regelungen zum Ausscheiden · Risiko Scheinselbstständigkeit

\section{Legal foundations of medical profession partnerships}

\section{Abstract}

In professional partnerships not only the premises of the practice, the practice furnishings and the nonmedical personnel are jointly utilized by two or more physicians, but medical personnel can also be jointly employed. As a rule, all income and expenditures are run jointly through the practice. Outwardly, the professional partnership forms a legal unit that is also reflected in the mutual liability of the partners. For the protection of this unit, the professional partnership is allowed to participate in the replacement of the licensing of a retiring partner through the successor, if the licensing is replaced within the professional partnership. Within the framework of the partnership contract, overall great care and in many aspects caution is also warranted. Additionally, the individual situation of the partnership and the individual partners must always be essentially taken into consideration. Even the local treatment situation has an important influence with respect to the requirements planning, particularly on regulation of retirement of a partner. In view of the complexity of the material, the partial aspects of the structure of a contract shown in this article and the appropriate regulatory options can only represent a small excerpt. In view of the legal overlaps from corporate law, laws concerning the medical profession and if necessary licensing laws, a lawyer with the appropriate experience and expertise should be commissioned with the construct of a medical partnership contract.

\section{Keywords}

Founding/start-up/takeover of a professional partnership - Structure of partnership agreements (joint practice contract) - Distribution of profits - Fair regulation of retirement - Risk of ostensible self-employment 


\section{Leistungsumfang}

Wichtig ist die Einigung darüber, welcher Gesellschafter welchen Beitrag insbesondere in zeitlicher Hinsicht leisten soll. Denn immer häufiger sind Gestaltungen, in denen etwa ein Gesellschafter Vollzeit arbeitet und ein anderer in Teilzeit (man denke nur an die vielen Gestaltungen zur Vereinbarkeit von Beruf und Familie). Auch die Themen Urlaub, Krankheit und Berufsunfähigkeit gehören in diesen weiten Bereich der Regelungserfordernisse. Gerade im Hinblick auf das Thema Berufsunfähigkeit bedarf es einer interessengerechten, im Ernstfall funktionierenden und auch im Hinblick auf die individuelle Gewinnverteilung durchdachten Regelung (Stichwörter: „Definition der Berufsunfähigkeit“ sowie „Feststellung bzw. Verpflichtung zur Überprüfung des Bestehens einer Berufsunfähigkeit“).

Ebenfalls hierher gehören Regelungen zur Nebentätigkeit, namentlich ob diese etwa grundsätzlich erlaubt sein sollen oder unter dem Vorbehalt der Genehmigung der Mitgesellschafter stehen, welche Folge die fehlende Genehmigung hat und wer die Kosten für die Nebentätigkeit trägt bzw. wem der Erlös hieraus zusteht. Auch die Frage, ob Nebentätigkeiten innerhalb oder nur außerhalb der Praxiszeiten und unter Nutzung der Praxisinfrastruktur erfolgen dürfen, bedarf zur Vermeidung von Streitigkeiten einer klaren Absprache.

\section{Entscheidungsfindung}

Soweit nicht das Gesetz vorschreibt, dass für eine Entscheidung der Gesellschaft die Zustimmung aller Gesellschafter erforderlich ist, obliegt es den Gesellschaftern zu regeln, wie die Gesellschafter ihre Beschlüsse fassen wollen. Dies betrifft einerseits die Art und Weise der Beschlussfassung (in einer gemeinsamen persönlichen Gesellschafterversammlung oder rein im Umlaufverfahren ohne persönliches Zusammentreffen) wie auch die Verteilung der Stimmen unter den Gesellschaftern und das für eine Entscheidung erforderliche Quorum. Hier besteht v. a. bei mehrgliedrigen Gesellschaften, also solchen, die aus drei und mehr Ge- sellschaftern bestehen, eine große Bandbreite an Gestaltungsmöglichkeiten, bei der aber auch immer Berücksichtigung finden muss, dass die Mitunternehmerinitiative der einzelnen Gesellschafter nicht $\mathrm{zu}$ stark reduziert wird.

Für den Fall, dass es zwischen den Gesellschaftern in Zukunft Streit gibt, wirkt sich dies in aller Regel auf die Beschlussfassung der Gesellschaft aus. Wichtig ist in diesem Zusammenhang daher, dass gerade im Streitfall allen Beteiligten klar ist, auf welchem Weg Entscheidungen getroffen werden können und binnen welcher Frist diese auf welchem Wege angegriffen werden können. Insbesondere ist durch klare Regelungen zu vermeiden, dass noch Monate nach Beschlussfassung durch querulatorische Widersprüche oder Klagen die Handlungsfähigkeit der Gesellschaft gefährdet wird.

\section{Verteilung von Gewinn und Verlust}

Auch wenn die Wahrscheinlichkeit eines Verlustes (d.h. es wird insgesamt weniger Umsatz erzielt, als für die reinen Kosten aufzubringen ist, sodass nach Abzug der Kosten kein Gewinn verbleibt, sondern sogar Schulden übrig bleiben) bei einer Arztpraxis jedenfalls bis zum Ausbruch der Covid-19-Pandemie immer als überaus gering galt, ist im Gesellschaftsvertrag zu regeln, wie ein hypothetischer Verlust $\mathrm{zu}$ verteilen wäre.

Deutlich mehr Gewicht wird aber natürlich in der Vertragsgestaltung auf die Verteilung des Gewinns gelegt. Die Gewinnverteilung ist in zahlreichen unterschiedlichen Gestaltungen möglich, von denen hier nur einige aufgezählt werden sollen:

- reine Verteilung nach Köpfen,

- reine Verteilung nach Beteiligung am Gesellschaftsvermögen,

- reine Verteilung nach Beteiligung an der erbrachten Arbeitsleistung in Zeit (fix oder dynamisch),

- reine Verteilung nach Beteiligung am Umsatz in Euro,

- reine Verteilung nach Beteiligung am Umsatz in Patientenzahlen,

- sog. Mischmodelle, in denen ein Sockelbetrag in Höhe einer bestimmten Prozentuale oder eines bestimmten Geldbetrages nach dem einen
Schlüssel verteilt wird (z. B. $60 \%$ des Gewinns entsprechend der Vermögensbeteiligung) und der darüber hinausgehende Teil nach einem anderen Schlüssel (z. B. die übrigen $40 \%$ leistungsabhängig nach der Beteiligung am Gesamtumsatz).

Es kann auch zugleich geregelt werden, unter welchen konkreten Bedingungen eine Anpassung der Gewinnverteilung stattzufinden hat. Ein Beispiel: Bei einer fixen Gewinnverteilung nach der erbrachten Arbeitsleistung in Zeit, bei der festgelegt ist, welcher Gesellschafter wie viele Stunden in der Woche arbeitet und daher welche Prozentuale vom Gewinn erhält, wird ergänzend vereinbart, dass bei einer Reduktion oder Aufstockung der Arbeitszeit eines Gesellschafters oder der faktischen Verschiebung der Verhältnisse der Arbeitsleistungen zueinander um einen bestimmten Faktor der Gewinnverteilungsschlüssel ebenfalls entsprechend angepasst wird.

Exkurs "Scheinselbstständigkeit“.Wichtig zu beachten ist, dass ein Gesellschafter einer Berufsausübungsgemeinschaft auch aufgrund seiner Stellung in der Gesellschaft als solcher sicher definiert werden kann. Bestehen in der Gesamtwertung der Rechte und Pflichten eines Gesellschafters Zweifel daran, ob dieser in angemessenem Umfang ein unternehmerisches Risiko trägt und unternehmerische Mitgestaltungsmöglichkeiten hat, kann es sein, dass er von den $\mathrm{Zu}$ lassungsbehörden, den Steuerbehörden oder den Sozialversicherungsträgern als Angestellter eingestuft wird. Es handelt sich dann um einen sog. ,Scheinselbstständigen“. Vor allem bei Zahnärzten war es jahrelang gang und gäbe, junge Nachwuchsärzte lediglich mit einem kleinen Prozentsatz (oder sogar gar nicht) am Vermögen der Gesellschaft zu beteiligen und ihnen anstelle einer Beteiligung am Gewinn einen Teil der von ihnen selbst erwirtschafteten Umsätze zuzugestehen. Das Problem hierbei ist, dass bei einer Beteiligung am Umsatz (anders als bei einer Beteiligung am Gewinn) kein unternehmerisches Risiko im Hinblick auf die Kosten und auch im Hinblick auf das Risiko eines theoretisch möglichen 
Verlustes besteht. Denn egal wie gut oder schlecht die Praxis insgesamt wirtschaftet, wird der Scheinselbstständige immer in gleicher Weise an seinen Umsätzen beteiligt. Die Höhe seiner Einkünfte ist also allein davon abhängig, wie viel er selbst erwirtschaftet, völlig losgelöst von der Praxis insgesamt. Die möglichen Konsequenzen einer solchen Scheinselbstständigkeit sind enorm. Denn bei einer Einstufung als Angestellter sind auf die Einkünfte in vollem Umfang Sozialabgaben $\mathrm{zu}$ entrichten, auch für die Vergangenheit. Zudem können Steuerbelastungen für die Vergangenheit entstehen. Und darüber hinaus ist v. a. bei einer Praxis, die (auch) gesetzlich krankenversicherte Patienten behandelt, die Auswirkung schnell existenzbedrohend. Denn die als Scheinselbstständiger erbrachten Leistungen, die der Arzt auf dem Papier als niedergelassener Arzt erbracht hat, während er in der rechtlichen Würdigung aber ein angestellter Arzt war, waren rückwirkend betrachtet nicht von seiner Abrechnungsgenehmigung als niedergelassener (zugelassener Arzt) gedeckt. Um vertragsärztliche Leistungen abrechnen zu können, hätte er eine Genehmigung als angestellter Arzt benötigt. Da ihm diese (logischerweise) fehlte, wurden sämtliche von diesem (scheinselbstständigen) Arzt erbrachten vertragsärztlichen Leistungen der Vergangenheit ohne Genehmigung abgerechnet und sind damit den Krankenversicherungen vollumfänglich $\mathrm{zu}$ erstatten. Dass diese Leistungen tatsächlich und durchaus auch von einem kompetenten Arzt erbracht wurden, ist in diesem Zusammenhang unerheblich. Sie wurden zulassungsrechtlich ohne Genehmigung erbracht, und daher sind die Kosten für diese Leistungen nicht von den gesetzlichen Versicherern $\mathrm{zu}$ tragen. Es drohen Regresse. Diese gehen schnell in Größenordnungen von mehreren Zehn- oder gar Hunderttausenden Euro.

\section{Vorsicht}

Nur wer im Hinblick auf seine mitunternehmerischen Chancen, Risiken, Mitsprache- und Gestaltungsmöglichkeiten in einer Gesamtschau als Gesellschafter angesehen werden kann, gilt steuerlich als Mitunternehmer, sozialversicherungsrechtlich als Selbständiger und zulassungsrechtlich als niedergelassener Arzt. Andernfalls kann er als Scheinselbstständiger, also Angestellter, gewertet werden.

\section{Besonders gefährlich}

Die einzelnen Rechtsgebiete sind insoweit unabhängig!

So kann es sein, dass ein Arzt beispielsweise steuerrechtlich als selbstständiger Gesellschafter anerkannt wird, aus Sicht der Zulassungsbehörden und/ oder Sozialversicherungsträger aber als Angestellter gewertet wird.

\section{Zuordnung von Arztstellen und Jobsharing-Konstellationen/ Jobsharing-Partnerschaften}

Sowohl im Hinblick auf die zulassungsrechtliche Situation wie auch auf die Verteilung des Gewinns sind Arztstellen oder bestimmte Jobsharing-Konstellationen bei (auch) vertragsärztlich tätigen Praxen zu regeln. $\mathrm{Zu}$ beachten ist hierbei, dass entgegen der früheren Handhabung angestellte Ärzte vertragsarztrechlich nicht mehr dem einzelnen Arzt zugeordnet sind, aus dessen Zulassung die Anstellungsgenehmigung generiert wurde, sondern der Berufsausübungsgemeinschaft. Hier kann es schnell zu Missverständnissen und Zerwürfnissen kommen, wenn die Eventualitäten nicht vorab beleuchtet, konsentiert und vertraglich festgehalten werden. Insbesondere gilt dies im Zusammenhang mit vertragsärztlichen Jobsharing-Partnerschaften. Zum einen hängt hier immer die vertragsärztliche Tätigkeit des sog. „Juniors"von der gemeinsamen Tätigkeit mit dem sog. "Senior“ ab, was bei den Ausscheidensregelungen besonders $\mathrm{zu}$ berücksichtigen ist. Zum anderen sollte geregelt werden, wie man damit umgeht, dass nach Ablauf von zehn Jahren oder im Zuge von bedarfsplanerischen Maßnahmen die Jobsharing-Zulassung in eine „normale“ Vollzulassung umgewandelt wird.

\section{Ausscheiden eines Gesellschafters}

Ein besonders weites Feld der rechtlichen Gestaltung eines Gesellschaftsvertrages machen die Regelungen zum Ausscheiden aus. Üblicherweise wird die im Gesetz verankerte Rechtsfolge, dass die Gesellschaft in bestimmten Fällen (z.B. Tod eines Gesellschafters, ordentliche und außerordentliche Kündigung, Eröffnung des Insolvenzverfahrens über das Privatvermögen eines Gesellschafters) aufgelöst wird, abbedungen durch eine sog. Fortführungsklausel. Diese besagt, dass der betreffende Gesellschafter ausscheidet und die übrigen Gesellschafter verpflichtet sind (stattdessen auch möglich: die reine Berechtigung ohne eine Verpflichtung), die Gesellschaft fortzuführen. Fehlt für einen solchen gesetzlich vorgesehenen Fall die Fortführungsklausel, kann es bei Eintritt dieses Falles zur ungewollten Auflösung der Gesellschaft kommen.

Hinsichtlich des Ausscheidens eines Gesellschafters sind verschiedene Aspekte $\mathrm{zu}$ berücksichtigen und miteinander in Einklang zu bringen. Vor allem sind die von Natur aus widerstreitenden Interessen der verschiedenen Betroffenen so aufzufangen, dass insgesamt ein Kompromiss entsteht, mit dem alle Beteiligten einverstanden sind und im Ernstfall ihre Existenz (oder ggf. die ihrer Erben) sichern können. Einerseits will man versetzt man sich in die Situation, selbst wegen Todes oder Berufsunfähigkeit auszuscheiden - gerne eine auskömmliche Abfindung für sich bzw. seine Erben. Andersherum will man aber - versetzt man sich in die Rolle des Verbleibenden im Fall des Versterbens oder der Berufsunfähigkeit eines anderen Gesellschafters - nicht mit immensen Abfindungszahlungen belastet sein, während man ja zugleich stark gefordert ist, die bisher gemeinsam geführte Praxis ganz alleine aufrechterhalten zu müssen bzw. kurzerhand einen geeigneten Nachfolger zu finden. In gleicher Weise hängt die Bewertung jedes denkbaren Ausscheidensgrundes (ordentliche Kündigung wegen Zerwürfnisses unter den Gesellschaftern, außerordentliche Kündigung wegen erheblicher Verfehlungen, Eröffnung des privaten Insolvenzverfahrens etc.) immer 
davon ab, ob man sich selbst in der Rolle des Ausscheidenden oder des Verbleibenden sieht. Die Vertragsgestaltung soll aber natürlich alle diese Szenarien jeweils für beide denkbaren Rollenverteilungen regeln. Besondere Bedeutung kommt im Zusammenhang mit dem Ausscheiden aus unterschiedlichen Gründen auch der interessengerechten Ausarbeitung eines Wettbewerbsverbotes zu. Da an ein solches einerseits sehr strenge Maßstäbe seitens der Rechtsprechung gelegt werden, $\mathrm{ihm}$ andererseits aber existenzielle Bedeutung bei der Fortführung der Praxis zukommen kann, ist auch und gerade diesbezüglich äußerste Sorgfalt geboten.

Gerade im Rahmen der ordentlichen Kündigung stellt sich bei Vertragsärzten maßgeblich die oft existentielle Frage: Zulassung mitnehmen oder dalassen? Darf der ausscheidende Gesellschafter seine Zulassung selbst an anderer Stelle weiter nutzen, kann er auf eine Abfindung für seine materielle Vermögensbeteiligung beschränkt werden. Soll der Ausscheidende aber verpflichtet werden, seine Zulassung durch die Gesellschaft nachbesetzen zu lassen, und überdies einem Wettbewerbsverbot unterliegen, das ihm verbietet, seine bisherigen Patienten in anderer Praxis weiter zu behandeln, so muss er hierfür natürlich eine angemessene Abfindung für seine Beteiligung am immateriellen Vermögen erhalten. Im letzteren Fall ist zudem für eine ausreichende Kündigungsfrist Sorge zu tragen, da die Gesellschaft die Zulassung nur dann gewinnbringend weiternutzen kann, wenn es ihr gelingt, innerhalb bestimmter Fristen einen Nachfolger einzubinden.

Immer wieder zu Streitigkeiten kommt es auch bei der Berechnung der Abfindung sowie der Praxisbewertung. Hier empfiehlt es sich, zumindest die Parameter der Bewertung festzulegen und $\mathrm{zu}$ regeln, durch wen eine Bewertung im Streitfall erfolgen soll.

Bei Neugründung einer Berufsausübungsgemeinschaft wie auch beim Eintritt in eine bestehende kann es sinnvoll sein, die ordentliche Kündigung für eine bestimmte Dauer auszuschließen, also eine Festlaufzeit des Gesellschaftsvertrages zu regeln.
Tritt ein Gesellschafter in eine bestehende Gesellschaft ein, kann es ratsam sein, Regelungen zur Erprobung zu treffen, so etwa die Vereinbarung, dass bei Ausscheiden des neu Eingetretenen innerhalb eines bestimmten Zeitraumes die Abfindung der Höhe nach auf den Betrag begrenzt ist, der als Kaufpreis für den zum Eintritt erworbenen Gesellschaftsanteil gezahlt wurde. Insgesamt stellt der Einstieg in eine bestehende Berufsausübungsgemeinschaft eine ganz andere rechtliche Herausforderung dar als die Neugründung einer Berufsausübungsgemeinschaft. Abzuraten ist grundsätzlich von der Übernahme eines bestehenden (in aller Regel auch im Hinblick auf die vorherige Situation bereits) veralteten Gesellschaftsvertrages, indem man durch einen schlichten Nachtrag hierzu den Beitritt in die Gesellschaft durch Übernahme der Gesellschaftsanteile regelt. Denn für die künftige Zusammenarbeit spielen die individuellen Belange eines jeden Gesellschafters eine Rolle. Am Beispiel des häufigsten Falles der altersbedingten Nachfolge: Die Situation des jungen Nachfolgers mit kleinen Kindern ist in jeder Hinsicht (finanziell, familiär, zeitlich, konditionell, fachlich) eine komplett andere als die des bisherigen Altgesellschafters. Es ist daher in keiner Weise interessengerecht, so zu tun, als würde sich außer im Hinblick auf den Namen des Gesellschafters nichts ändern. Der Gesellschaftsvertrag ist hier an die veränderte Situation anzupassen. Umso mehr gilt dies, wenn der bestehende Gesellschaftsvertrag für eine ganz andere Grundkonstellation gestaltet wurde, z. B. der Vertrag noch von einer Gesellschaft mit nur 2 Gesellschaftern ausgeht, der aber inzwischen ein dritter hinzugetreten ist (oder andersherum).

\section{Anpassung an die individuellen Gegebenheiten}

Bei der Ausarbeitung des Gesellschaftsvertrages gibt es in vielen Punkte sehr unterschiedliche Gestaltungsmöglichkeiten. Wichtig ist insoweit, die konkrete Situation vollständig zu berücksichtigen, etwa die Anzahl der Gesellschafter, den persönlichen und fachlichen Hintergrund und die familiäre Situation des Einzelnen (eigenes Alter, Anzahl der Kinder und deren Alter etc.), aber auch die örtliche Versorgungssituation. Zudem müssen die Regelungen der einzelnen Aspekte wiederum miteinander in Einklang stehen. So muss (um nur zwei Beispiele zu nennen) die Regelung zur Kostentragung bei Krankheit und Berufsunfähigkeit mit der Regelung zur Gewinnverteilung harmonieren. Das Wettbewerbsverbot wiederum ist auf die konkreten Ausscheidensregelungen im Hinblick auf die Zulassung und die Abfindung abzustimmen.

\section{Fazit für die Praxis}

- Zum Zeitpunkt der Gründung oder des Beitritts in eine Berufsausübungsgemeinschaft erscheinen die aus anwaltlicher Sicht zwingend erforderlichen Regelungen den Beteiligten oft als überflüssig oder unnötig kompliziert. Hier ist aber immer zu bedenken, dass Sie einen Vertrag nicht für die Zeit verfassen, in der sich alle verstehen und vertragen und sich einig sind, wie welche Dinge zu handhaben sind. Einen Vertrag benötigen Sie immer genau dann, wenn Uneinigkeiten oder gar Streit entstehen. Und dann benötigen Sie Regelungen, die für jeden Fall eindeutig und klar regeln, wie zu verfahren ist. So vermeidet jeder friedlich im Vorfeld getroffene Konsens einen unnötigen weiteren Kriegsschauplatz im Streitfall.

- Insgesamt bleibt daher festzuhalten, dass es einer versierten und sorgfältigen anwaltlichen Ausarbeitung bedarf, um die konkreten und individuellen Gegebenheiten des Einzelfalles interessengerecht umzusetzen und in den verschiedenen denkbaren Szenarien weitest möglich zu wahren. Da dies angesichts der Verschiedenartigkeit der teilweise widerstreitenden Interessen der Beteiligten teilweise der "Quadratur des Kreises" gleichkommt, bedarf es zudem einer anwaltlichen Betreuung zur Begleitung und Moderation der gemeinsamen Konsensfindung, die nicht konfrontativ wirkt, sondern die 
Beteiligten konstruktiv zusammenführt.

\section{Korrespondenzadresse}

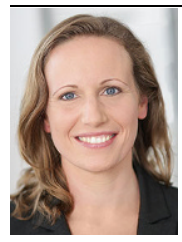

\section{Vera Keisers}

Kanzlei am Ärztehaus

Dorpatweg 10, Germania

Campus, 48159 Münster,

Deutschland

v.keisers@kanzlei-am-

aerztehaus.de

\section{Einhaltung ethischer Richtlinien}

Interessenkonflikt. V. Keisers ist angestellte Rechtsanwältin der Kanzlei am Ärztehaus, Justitiarin der GÄCD.

Für diesen Beitrag wurden vom Autor keine Studien an Menschen oder Tieren durchgeführt.

\section{September 2020}

Hamburg 18.-20.09.2020

35. Jahrestagung der Deutschen Gesellschaft für Dermatochirurgie e.V.

Themen: Akne inversa, Filler und Augmentation,

Licht und Laser, Liposuktion u.v.m.

Auskunft: Frau Conventus Congressma Julia

Wrenczycki, Conventus Congressmanagement \&

Marketing GmbH, Carl-Pulfrich-Str. 1, 07745 Jena,

Fon: 036413116390 ,

conferencemarketing@conventus.de

Ulm 23.-24.09.2020

27. Kurs in funktionell-ästhetischer

Rhinochirurgie

Themen: Funktionell ästhetische Rhinochirurgie,

OP-Kurs mit Übungen an Fresh-Frozen Präparaten

Wiss. Leitung: Prof. Dr. M. O. Scheithauer,

Gastdozent: Prof. Dr. J. Kainz, Graz

Auskunft: Janka Steiner, Universitätsklinikum UIm, Frauensteige 12, $89075 \mathrm{UIm}$

Fon: 0731/50059505,

hno.kurs@uniklinik-ulm.de,

https://www.uniklinik-ulm.de/hals-nasen-und-

ohrenheilkunde.html

\section{April 2021}

Stuttgart 21.-22.04.2021

28th Stuttgart Advanced Course for Rhinoplasty with Live Surgical Demonstration

Themen: Analysis of Primary \& Secondary Cases, Development of Specific Surgery Concepts, Video presentations

Wiss. Leitung: Dr. S. Haack und

Prof. Dr. Dr. h. c. W. Gubisch, Stuttgart

Auskunft: boeld communication $\mathrm{GmbH}$

Kapuzinerstr. 9d, 80337 München,

congress@bb-mc.com,

http://www.stuttgart-rhinoplasty-2021.de/

Stuttgart 23.-24.04.2021

3rd Stuttgart Symposium on Nasal

Reconstruction with Live Surgery

Themen: Reconstruction of lining, framework

and cover

Auskunft: boeld communication $\mathrm{GmbH}$

Kapuzinerstr. 9d, 80337 München,

congress@bb-mc.com,

http://www.stuttgart-rhinoplasty-2021.de/ 